\title{
Genome-wide association study and polygenic risk score analysis for hearing measures in children
}

\section{Judith Schmitz $\odot$ | Filippo Abbondanza | Silvia Paracchini ${ }^{(0)}$}

School of Medicine, University of St Andrews, St Andrews, UK

\section{Correspondence}

Silvia Paracchini, School of Medicine, University of St Andrews, St Andrews, UK. Email: sp58@st-andrews.ac.uk

\section{Funding information}

Deutsche Forschungsgemeinschaft, Grant/ Award Number: SCHM 3530/1-1 (418445085); Royal Society, Grant/Award Number: UF150663; UK Medical Research Council and Wellcome, Grant/Award Number: 217065/Z/19/Z; Wellcome Trust, Grant/ Award Number: 105621/Z/14/Z

\begin{abstract}
An efficient auditory system contributes to cognitive and psychosocial development. A right ear advantage in hearing thresholds (HTs) has been described in adults and atypical patterns of left/right hearing threshold asymmetry (HTA) have been described for psychiatric and neurodevelopmental conditions. Previous genome-wide association studies (GWASs) on HT have mainly been conducted in elderly participants whose hearing is more likely to be affected by external environmental factors. Here, we investigated HT and HTA in a children population cohort (ALSPAC, $n=6,743$ ). Better hearing was associated with better cognitive performance and higher socioeconomic status. At the group level, HTA suggested a left ear advantage (mean $=-0.28 \mathrm{~dB}$ ) that was mainly driven by females. SNP heritability for HT and HTA was 0.13 and 0.02 , respectively $(n=4,989)$. We found a modest negative genetic correlation between $\mathrm{HT}$ and reading ability. GWAS for HT ( $n=5,344)$ did not yield significant hits but polygenic risk scores for higher educational attainment (EA, $B=-1,564.72, p=.008$ ) and schizophrenia $(B=-241.14$, $p=.004$ ) were associated with lower $\mathrm{HT}$, that is, better hearing. In summary, we report new data supporting associations between hearing measures and cognitive abilities at the behavioral level. Genetic analysis suggests shared biological pathways between cognitive and sensory systems and provides evidence for a positive outcome of genetic risk for schizophrenia.
\end{abstract}

KEYWORDS

ALSPAC, audiometry, cognition, laterality, neurodevelopment

\section{1 | INTRODUCTION}

The auditory system involves a series of complex distributed cerebral networks and its impairment affects psychosocial, emotional, and cognitive development (Stevenson et al., 2018). Hearing-impaired children are at increased risk for learning disabilities (S. M. R. Choi, Kei, \& Wilson, 2020) and even within the normal range, better hearing has been associated with better reading skills, working memory and nonverbal IQ in a sample of 1,638 UK school children (Moore, Zobay, \& Ferguson, 2020).
Hearing ability is usually defined as the threshold in decibel (dB) at which a tone is perceived, so that lower values indicate better hearing. An age-related hearing decline is well documented. In a Korean general population sample ( $n>15,000$ ), the hearing threshold (HT) for medium frequencies increased from $3 \mathrm{~dB}$ in adolescents to $38 \mathrm{~dB}$ in elderly participants (Park, Shin, Byun, \& Kim, 2016). A sex difference in favor of women was found in adults ( $n=10,145$; 3069 years old), but not in children and young adults ( $n=3,458)$.

Successful hearing requires transformation of changes in air pressure into vibrations in the basilar membrane that is transferred onto 
sensory hair cells of the inner ear, whose depolarization is initiated by deflection of mechano-sensitive hair bundles (Schwander, Kachar, \& Müller, 2010). The auditory nerve transmits these signals to the cochlear nucleus in the brainstem. The majority of the input is transmitted to the contralateral superior olivary complex, while a minor part of the input is transmitted ipsilaterally (Felix, Gourévitch, \& Portfors, 2018; Warren \& Liberman, 1989). Greater contralateral medial olivonuclear suppression in the right compared to the left ear (Khalfa \& Collet, 1996) has been suggested as the underlying correlate of a fundamental functional asymmetry between the left and right ear. This hearing threshold asymmetry (HTA) has typically been reported as $\mathrm{HT}$ left-HT right so that positive values indicate an advantage of the right and negative values indicate an advantage of the left ear. In a study of more than 50,000 adults, Chung, Mason, Gannon, and Willson (1983) reported a right ear advantage (HTA between 1 and $4 \mathrm{~dB}$ ) with more pronounced HTA in males than in females. In 1,191 children, a right ear advantage has been reported, albeit to a smaller extent than in adults (Eagles, 1973). Other authors found a general right ear advantage in males ( $n=\sim 400$, HTA between 0.1 and $0.5 \mathrm{~dB}$ ) and a left ear advantage in females for specific frequencies ( $n=\sim 400$, HTA between -0.1 and -0.4 dB) (Kannan \& Lipscomb, 1974). Smaller studies reported a general left ear advantage in children (Rahko \& Karma, 1989; Roche, Siervogel, Himes, \& Johnson, 1978).

An absence of HTA has been reported in schizophrenia (Gruzelier \& Hammond, 1979; Mathew, Gruzelier, \& Liddle, 1993) and ADHD (Combs, 2002). Moreover, symmetrical contralateral suppression in the olivary complex in the left and the right ear in schizophrenia (Veuillet et al., 2001) is in contrast with the right ear advantage typically found in controls. In children and adolescents, a right ear advantage has been reported in a sample of $n=22$ with autism spectrum disorder (ASD) while no asymmetry was found in the control group (Khalfa et al., 2001). A developmental effect toward stronger HTA has been reported in controls that was absent in ASD children ( $n=24$ ) (James \& Barry, 1983). Reduced laterality in processing auditory stimuli was reported in ASD and bipolar disorder (BIP) suggesting that HTA is linked to neurodevelopmental disorders (Reite et al., 2009; Schmidt, Rey, Oram Cardy, \& Roberts, 2009).

Twin and family studies estimated the heritability for HT to range from 0.26 to 0.75 with larger environmental effects for the nondominant ear (Gates, Couropmitree, \& Myers, 1999; Viljanen et al., 2007; Viljanen et al., 2007). Genome-wide association studies (GWASs) focused on age-related hearing loss in subjects ranging from 45 to 75 years of age and identified genes including GRM7 and ESRRG (Fransen et al., 2015; Friedman et al., 2009; Huyghe et al., 2008; Nolan et al., 2013). GWAS for normal hearing included subjects from 18 to 92 years of age (Girotto et al., 2011; Vuckovic et al., 2015; Wolber et al., 2014) and implicated several genes (i.e., DCLK1, PTPRD, GRM8, CMIP, SIK3, PCDH20, SLC28A3), some of which have been associated with neurodevelopmental traits (Håvik et al., 2012; Newbury et al., 2009; Newbury et al., 2019; Scerri et al., 2011; Takaki et al., 2004; Zhang et al., 2014). A case control design based on electronical health records on age-related hearing loss identified SNPs near ISG20 and TRIOBP (Hoffmann et al., 2016), which had previously been associated with prelingual nonsyndromic hearing loss
(Diaz-Horta et al., 2012). In the UK Biobank, 41 and 7 independent loci have been identified for hearing difficulty and hearing aid use, respectively, implicating genes such as CDH23, EYA4, KLHDC7B, and again TRIOBP (Wells et al., 2019). Mutations in $C D H 23$ have been associated with early-onset hearing loss and Usher syndrome causing early-onset deafness (Schultz et al., 2011).

However, older subjects have had more exposure to environmental factors, which might affect hearing, such as extensive noise (Stanbury, Rafferty, \& Rosenman, 2008), medication (Ruhl, Cable, \& Martell, 2014), chemicals (Morata, 2003), and medical conditions (Kakarlapudi, Sawyer, \& Staecker, 2003). An investigation of HT in 250 monozygotic (MZ) and 307 dizygotic (DZ) twin pairs from 36 to 80 years of age suggests that environmental effects become more significant with age (Karlsson, Harris, \& Svartengren, 1997). Despite this age effect, no study has ever investigated genetic factors involved in hearing function in children.

Here, we analyzed hearing measures in children from the Avon Longitudinal Study of Parents and Children (ALSPAC) ( $n=6,743$ ). Consistent with previous studies, we found that better hearing is associated with enhanced cognitive skills and higher socioeconomic status (SES). We report the first GWAS for HT in children $(n=5,344)$. In addition to single marker trait associations, we conducted genebased and gene set analysis and tested the effects of polygenic risk scores (PRS) for a range of neurodevelopmental disorders, IQ, and educational attainment (EA). Our results suggest that PRS for higher EA and schizophrenia are associated with better hearing.

\section{2 | MATERIALS AND METHODS}

\subsection{Cohort}

ALSPAC is a longitudinal cohort representing the general population living in the Bristol area. Pregnant women resident in the county of Avon, United Kingdom, with expected dates of delivery from April 1,1991 , to December 31,1992, were invited to take part in the study, resulting in 14,062 live births and 13,988 children who were alive at 1 year of age (Boyd et al., 2013; Fraser et al., 2013). From age 7, all children were invited annually for assessments on a wide range of physical, behavioral and neuropsychological traits. Informed written consent was obtained from the parents after receiving a complete description of the study at the time of enrolment into ALSPAC, with the option to withdraw at any time. Ethical approval for the present study was obtained from the ALSPAC Law and Ethics Committee and the Local Research Ethics Committees. The ALSPAC study website contains details of all the data that is available through a fully searchable data dictionary (http://www.bris.ac.uk/alspac/researchers/dataaccess/data-dictionary/).

\section{2 | Phenotypes}

Audiometry was performed according to British Society of Audiologists standards. Hearing tests were carried out in a room with minimal external noise. Testing was stopped if the background noise level 
exceeded $35 \mathrm{dBA}$. The air conduction threshold, that is, the lowest intensity in decibels at which a tone is perceived $50 \%$ of the time ( $\mathrm{dBHL}$, decibel hearing level), was tested using either a GSI 61 clinical audiometer or a Kamplex AD12 audiometer. Lower $\mathrm{dBHL}$ values indicate better hearing. For each ear, the air conduction threshold level was tested at $500 \mathrm{~Hz}, 1 \mathrm{kHz}, 2 \mathrm{kHz}$, and $4 \mathrm{kHz}$. For each frequency, stimuli were first presented on the right and then on the left ear. The average threshold across different frequencies was derived for each ear.

After applying exclusion criteria (Appendix S1), a sample of $n=6,743$ was available for phenotypic analysis (3,344 females, 3,391 males, 8 missing values for sex, mean age $=7.59$ years, $\mathrm{SD}=0.32$ years).

HT was defined as the average air conduction threshold on the better ear. HTA was defined as the absolute difference in air conduction threshold between the left and right ear. Thus, positive values indicate a right ear advantage, while negative values indicate a left ear advantage. Handedness was assessed in terms of writing hand $(5,805$ right-handers, 787 left-handers, and 151 missing values).

Cognitive skills were assessed using tests for reading ability (Rust, Golombok, \& Trickey, 1993), communication skills (Bishop, 1998), listening comprehension (Rust, 1996), short-term memory (Gathercole, Willis, Baddeley, \& Emslie, 1994), total IQ, verbal IQ, performance IQ (Wechsler, Golombok, \& Rust, 1991), and EA measured as capped General Certificate of Secondary Education (GCSE) scores (for detailed descriptions, see Appendix S1). Maternal highest educational qualification during pregnancy was used as a proxy for SES (Rashid et al., 2018). Educational qualification was grouped into "CSE and no education," "Vocational," "O level," "A level," and "Degree."

Children were assigned to neurodevelopmental and control subgroups as defined for the ALSPAC sample previously (Scerri et al., 2011). We specified subgroups for language impairment (LI) ( $n=155)$, reading disability (RD) ( $n=141)$, ASD $(n=35)$, ADHD $(n=21)$, comorbidity ( $n=49$ ), and a control sample matched for sex $(n=2,071)$. The strategies for subgroup assignments are reported in the Appendix S1.

\section{3 | Genotype quality control (QC) and imputation}

Genotypes were generated on the Illumina HumanHap550-quad array at the Wellcome Trust Sanger Institute, Cambridge, United Kingdom, and the Laboratory Corporation of America, Burlington, NC, United States. Standard QC was performed as described elsewhere (Brandler et al., 2013). Population stratification was assessed by multidimensional scaling analysis and compared with Hapmap II (release 22) European descent, Han Chinese, Japanese, and Yoruba reference populations. All individuals with non-European ancestry were removed. In total, 9,115 subjects and 500,527 SNPs passed QC filtering. Haplotypes were estimated using ShapelT (v2.r644). QC-filtered autosomal SNPs were imputed using Impute v3 using the HRC 1.1 reference data panel. Poorly imputed SNPs (Info score $<0.8$ ) and SNPs with low minor allele frequency $(\mathrm{MAF}<0.05)$ were excluded from further analysis.

\subsection{Statistical analysis}

\subsection{1 | SNP heritability}

Genome-wide genotype data were available for 5,344 children with phenotypes (2,691 males, 2,653 females, mean age $=7.58$ years, $\mathrm{SD}=0.31$ years). HT and HTA were inverse rank-transformed to achieve a normal distribution. We estimated SNP heritability (SNP $\left.h^{2}\right)$ using genome-based restricted maximum-likelihood (GREML) analysis implemented in GCTA (Genome-wide complex trait analysis) (Yang, Lee, Goddard, \& Visscher, 2011), which compares phenotypic similarity and genotypic similarity based on a genetic-relationship matrix (GRM) (Yang et al., 2010). We created a GRM based on directly genotyped SNPs and including unrelated individuals $(n=4,989$; identity by descent $[\mathrm{IBD}]<0.05)$. SNP $h^{2}$ was estimated on the basis of this GRM using sex and the first two principal components as covariates. Principal components were calculated based on directly genotyped (MAF < 0.05) and LD pruned $\left(r^{2}<0.01\right.$ within a $50 \mathrm{~kb}$ window) SNPs (excluding high LD regions) using Plink v2 (Chang et al., 2015).

\subsection{2 | Genetic correlation analysis}

We used bivariate GREML to estimate genetic correlations $\left(r_{g}\right)$ between $\mathrm{HT}$ and the eight cognitive skills used for behavioral analysis based on the GRM described above ( $n=4,989$ unrelated children) and using sex and the first two principal components as covariates.

\subsection{3 | GWAS}

Association testing was performed using BOLT-LMM v2.3.4 (Loh et al., 2015) under the standard infinitesimal linear mixed model (LMM) framework specifying sex and the first two principal components as covariates. Since BOLT-LMM allows for the inclusion of related individuals (Loh, Kichaev, Gazal, Schoech, \& Price, 2018), GWAS was run on the whole available sample with phenotypes and genotypes ( $n=5,344$ ). Overall, 5,305,352 SNPs that were either directly genotyped or imputed and passed QC were tested for association. The genomic inflation factor $(\lambda)$ was calculated for all SNPs and revealed no evidence of population structure (HT: $\lambda=1.02$, HTA: $\lambda=1.00$ ).

\subsubsection{Annotation and gene mapping}

We applied FUMA v1.3.6a (Watanabe, Taskesen, van Bochoven, \& Posthuma, 2017) on the GWAS summary statistics. Functional consequences of SNPs were obtained by performing ANNOVAR (Wang, Li, \& Hakonarson, 2010) using Ensembl genes (build 92). SNPs were mapped to genes based on positional mapping. Intergenic SNPs were annotated to the closest genes upstream and downstream. Input SNPs were mapped to 18,360 protein-coding genes. 


\subsection{5 | Replication analysis}

For HT, we specifically tested for replication of SNPs associated with quantitative hearing phenotypes in previous studies $\left[p<10^{-5}\right.$ (Fransen et al., 2015; Girotto et al., 2011), $p<10^{-6}$ (Vuckovic et al., 2015; Wolber et al., 2014)]. We also tested for replication of SNPs showing genome-wide significance in case-control GWAS on age-related hearing loss [ $p<5 \times 10^{-8}$ (Hoffmann et al., 2016; Wells et al., 2019)]. This procedure resulted in 644 SNPs, which we selected as predefined lead SNPs in FUMA. Of the 644 SNPs, FUMA identified 272 independent SNPs ( $r^{2} \leq 0.6$, default setting), which were used to determine boundaries of LD blocks that encompassed a total of 60 loci including 1,126 SNPs ( $r^{2} \geq 0.6$, default setting). Among these 1,126 SNPs, 411 (representing all 60 loci) were available in our study, resulting in a Bonferroni-corrected significance level of $.05 / 60=8.3 \times 10^{-4}$.

\subsection{6 | Gene-based and gene set analyses}

FUMA implements MAGMA v1.08 (de Leeuw, Mooij, Heskes, \& Posthuma, 2015) to summarize SNP associations at the gene level (gene-based analysis) and associate the set of genes to biological pathways (gene set analysis). Gene-based $p$-values were computed using an F-test in a multiple linear principal components regression while accounting for LD between SNPs. Genome-wide significance was defined as $p=.05 / 18,360=2.7 \times 10^{-6}$. For gene set analysis, MAGMA converts gene-based $p$-values into $z$ values, which reflect the strength of association. MAGMA performs a competitive gene set analysis, comparing the mean association of genes within a gene set with the mean association of genes not in the gene set while correcting for gene size and density. Gene set $p$-values were computed for 7,343 gene ontology (GO) terms for biological processes obtained from MsigDB v5.2. The Bonferroni-corrected significance level was set to $.05 / 7,343=6.8 \times 10^{-6}$.

\subsection{7 | PRS}

PRS analyses were carried out using PRSice 2.3.3 (S. W. Choi \& O'Reilly, 2019). PRSice uses GWAS summary statistics as training GWAS to build PRS, which are then tested as predictors for the phenotype of interest in the target sample (ALSPAC). Psychiatric Genomics Consortium summary statistics were downloaded (https://www.med. unc.edu/pgc/data-index/) for schizophrenia (Schizophrenia Working Group of the Psychiatric Genomics Consortium, 2014), ADHD (Neale et al., 2010), ASD (Autism Spectrum Disorders Working Group of The Psychiatric Genomics Consortium, 2017), and BIP (Psychiatric GWAS Consortium Bipolar Disorder Working Group, 2011) as these are the psychiatric and neurodevelopmental conditions often found to be associated with reduced laterality (Khalfa et al., 2001; Mathew et al., 1993; Reite et al., 2009; Veuillet et al., 2001). Based on associations of HT with cognitive skills, GCSE scores, and SES, we downloaded summary statistics for IQ (Savage et al., 2018) from the Complex Trait Genetics lab website (https://ctg.cncr.nl/software/summary_statistics) and EA (Lee et al., 2018) from the Social Science Genetic Association Consortium (https://www.thessgac.org/data).

SNPs were clumped based on LD $\left(r^{2} \geq 0.1\right)$ within a $250 \mathrm{~kb}$ window. PRS were derived as the weighted sum of risk alleles based on odds ratios or beta values from the training GWAS summary statistics. Sex and first two principal components were included as covariates. Results are presented for the optimal training GWAS $p$-value threshold (explaining the highest proportion of phenotypic variance in $\mathrm{HT}$ ) as well as GWAS $p$-value thresholds of .001, .05, .1, .2, .3, .4, .5, and 1 (all SNPs included). For optimal training GWAS $p$-value thresholds and number of SNPs included in the PRS, see Table S1. For six training GWAS, the Bonferroni-corrected significance level was set to $.05 / 6=.0083$.

Data preparation and visualization was performed using R v.4.0.0. All analysis scripts are available through Open Science Framework (https://osf.io/gewj2/).

\section{3 | RESULTS}

\section{1 | Phenotypes}

We first analyzed the distribution of HT and HTA in the overall sample ( $n=6,743$ ) (results for the subset used in the GWAS, $n=5,344$, are shown in Figure S1). HT ranged from -8.75 to 20.00. Mean HT was $6.14(\mathrm{SD}=3.97$ ) (Figure $1(\mathrm{a})$ ). Females $(n=3,344)$ showed slightly higher HT (mean $=6.30, \mathrm{SD}=4.06)$ than males $(n=3,391$, mean $=5.98, \mathrm{SD}=3.88$ ), $t_{(6709.2)}=-3.31, p=.001$ (Figure 1(b)).

HTA ranged from -18.75 to 20 and revealed a significant left ear advantage for the overall sample (mean $=-0.28, S D=3.75$ ) as determined by one-sample $t$-test against zero $\left(t_{(6742)}=-6.17\right.$, $\left.p=7.37 \times 10^{-10}\right) \quad($ Figure $1(\mathrm{C}))$. Females showed stronger HTA (mean $=-0.48, \mathrm{SD}=3.81$ ) than males (mean $=-0.09, \mathrm{SD}=3.68$ ), $t_{(6716.9)}=4.33$., $p=1.54 \times 10^{-5}$ (Figure $1(D)$ ). We thus performed one-sample $t$-tests against zero for females and males separately. While females showed a significant left ear advantage $\left(t_{(3343)}\right.$ $\left.=-7.30, p=3.50 \times 10^{-13}\right)$, there was no ear advantage in males $\left(t_{(3390)}=-1.37, p=.172\right)$. Linear regression revealed no evidence for an effect of age on HT $\left(F_{(1,6741)}=2.86, \beta=-0.005, p=.091\right)$ or HTA $\left(F_{(1,6741)}=3.61, \beta=-0.005, p=.058\right)$. Similarly, there was no evidence for an effect of handedness on HTA (right-handers: $n=5,805$, mean $=-0.28, \mathrm{SD}=3.75$; left-handers: $n=787$, mean $=-0.24$, $\left.\mathrm{SD}=3.72, \mathrm{t}_{(1015)}=-0.29, p=.776\right)$.

Bivariate Pearson correlations revealed significant positive correlations among the different cognitive traits as previously reported (Scerri et al., 2011). There were significant negative correlations after conservative Bonferroni correction (45 comparisons, $p<.0011$ ) for all cognitive traits but listening comprehension with HT (Figure 2), indicating that lower HT (better hearing) is associated with better cognitive performance (correlation plots are shown in Figure S2). There was no association between HTA and cognitive traits.

One-way between-subjects ANOVAs were conducted to assess the effect of SES on HT and HTA. There was a significant effect of SES on $\mathrm{HT}\left(F_{(4,6137)}=7.25, p=8.1 \times 10^{-6}\right)$. Post hoc comparisons 
(a)

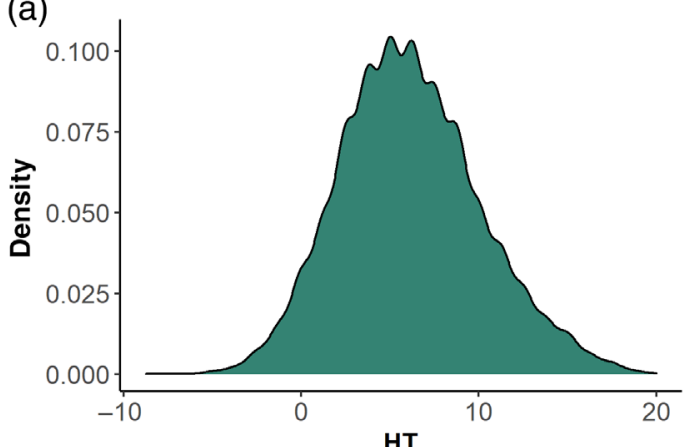

(c)

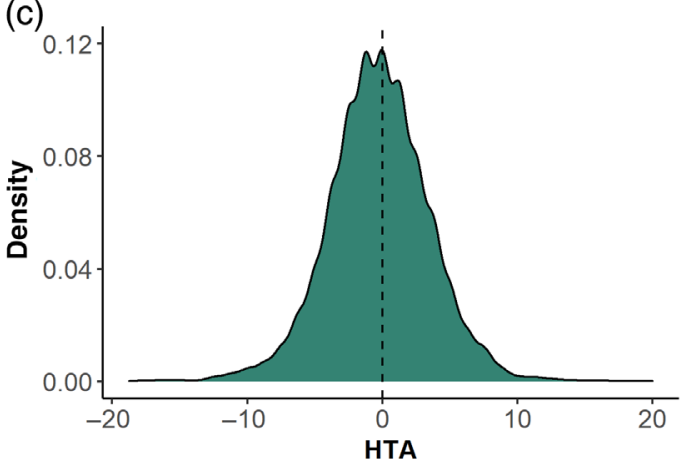

(b)

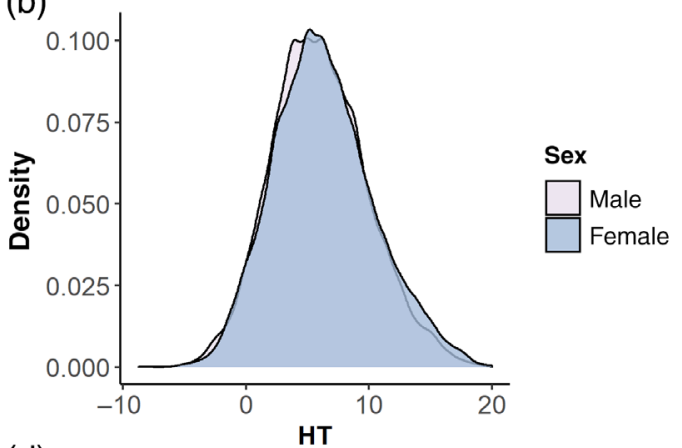

(d)

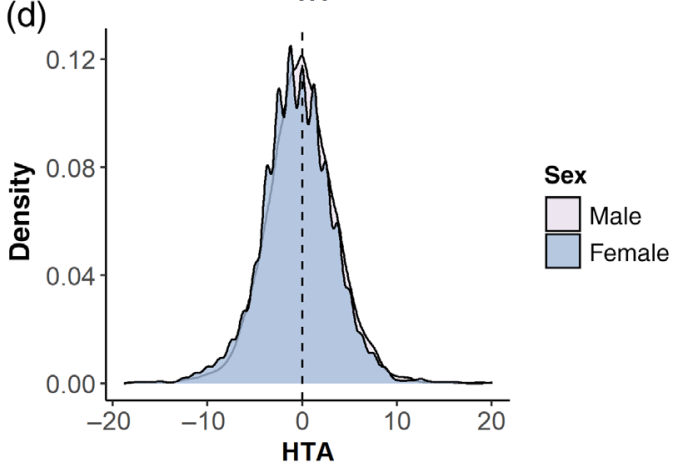

FIGURE 1 Distribution of HT and HTA. (a) Distribution of HT (better ear) in the overall sample ( $n=6,743)$, and (b) as a function of sex. (c) Distribution of HTA in the overall sample, and (d) as a function of sex. The dotted line represents no asymmetry between the left and right ear [Color figure can be viewed at wileyonlinelibrary.com]

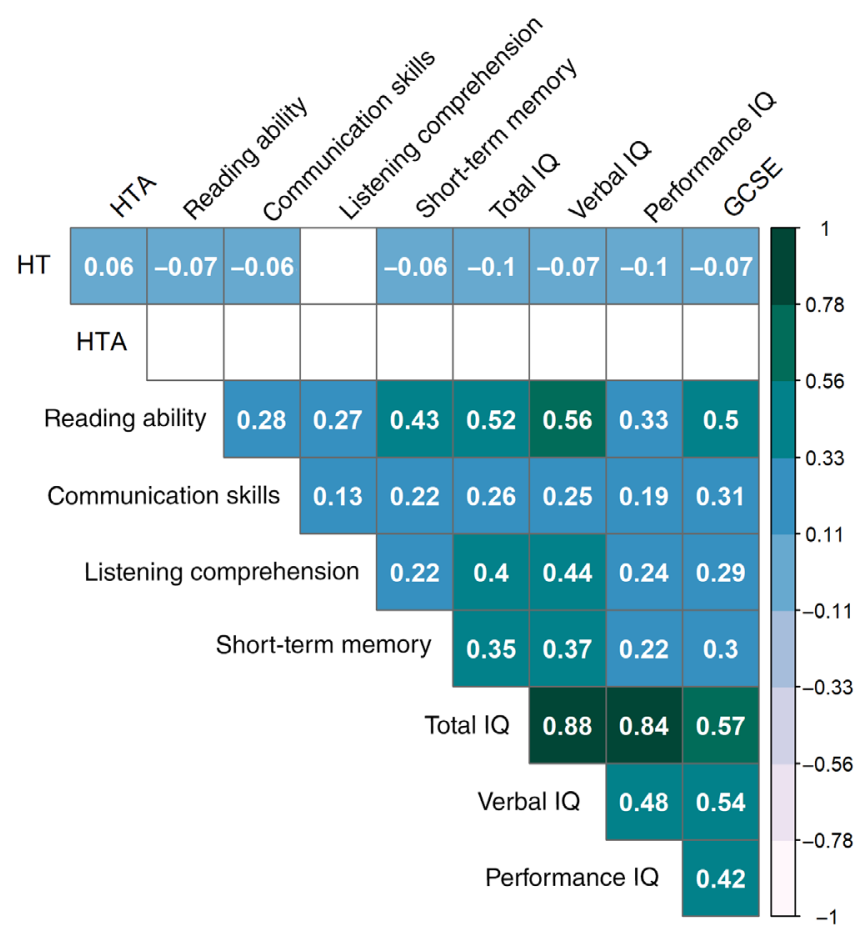

FIGURE 2 Correlation matrix for HT, HTA, and cognitive traits. Correlation coefficients are shown if passing the Bonferroni-corrected significance level $(p<.0011)$. Sample sizes range from $n=4,730$ to $n=6,743$ depending on data availability [Color figure can be viewed at wileyonlinelibrary.com] using the Tukey test indicated significantly lower HT for "O level" (mean $=6.12$, SD = 3.95), "A level" (mean $=5.91$, SD $=3.93$ ), and "Degree" (mean $=5.92, \mathrm{SD}=4.05)$ compared to "CSE" (mean = 6.69, $\mathrm{SD}=4.12$ ) and significantly lower HT for "A level" and "Degree" compared to "Vocational" (mean $=6.52, \mathrm{SD}=3.84$ ), indicating that higher SES is associated with better hearing (Figure S3). There was no significant effect of SES on HTA $\left(F_{(4,6137)}=1.78, p=.130\right)$.

Two-sample $t$-tests revealed no difference between children affected by neurodevelopmental disorders and sex-matched controls in HT (Table S2) or HTA (Table S3). However, there was a consistent pattern across neurodevelopmental subgroups with more negative HTA compared to the control group, indicating more leftward asymmetry.

\section{2 | SNP heritability}

In $n=4,989$ unrelated children, SNP $h^{2}$ was 0.13 (SE $=0.07$ ) for HT and 0.02 (SE $=0.06$ ) for HTA. Because of the extremely low SNP $h^{2}$ for HTA, subsequent genetic analyses were only performed for HT.

\section{3 | Genetic correlation}

HT showed a trend toward negative genetic correlation with reading ability $\left(r_{g}=-.34, \mathrm{SE}=0.23\right.$, Figure 3$)$, indicating a small degree of 


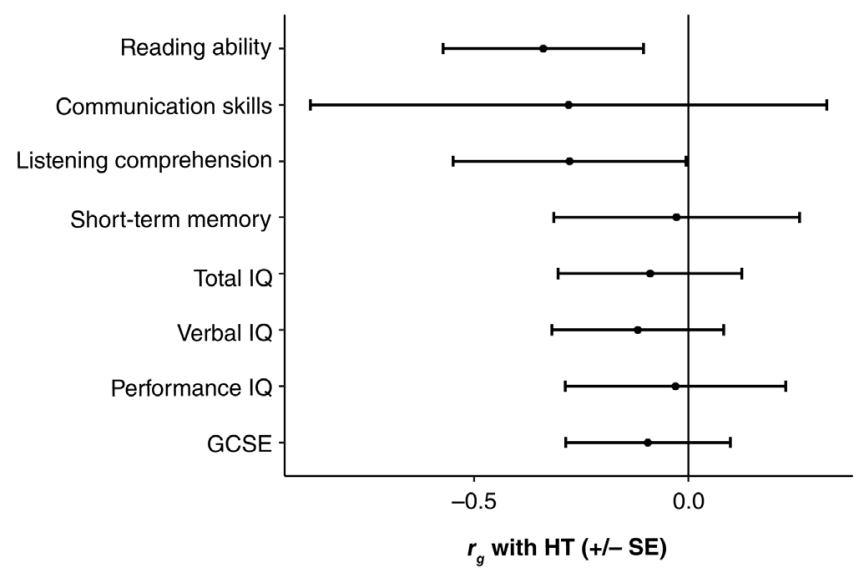

FIGURE 3 Results of genetic correlation $\left(r_{g}\right)$ analysis between HT and cognitive traits

common variant heterogeneity between the two traits. All other genetic correlations were nonsignificant.

\section{\begin{tabular}{l|l}
3.4 & GWAS
\end{tabular}}

GWAS was performed in $n=5,344$ children. No genome-wide significant results were observed on the level of SNPs (Table S4) or on the level of genes. Manhattan and QQ plots are shown in Figures S4-S7.

In the replication analysis of SNPs derived from previous literature, one locus (including six SNPs located in an intron of the CCBE1 gene) reached Bonferroni-corrected significance (Table S5). The lead SNP ( $r$ 12955474, $B=0.17, p=8.1 \times 10^{-5}$ ) had been previously reported in a GWAS for the third $\mathrm{PC}$ on $\mathrm{HT}$ over different frequencies $(0.25,0.5,1,2,3,4,6,8 \mathrm{kHz})\left(B=-0.10, p=3.6 \times 10^{-7}\right)$ (Fransen et al., 2015).

In gene set enrichment analysis, no GO term reached the Bonferroni-corrected level of significance $\left(p=6.8 \times 10^{-6}\right)$ for HT. "Response to interleukin 12 (GO: 0070671)" ( $p=1.5 \times 10^{-5}$ ) was the strongest association.

Since there was no significant SNP $h^{2}$ for HTA, we did not perform genetic correlation or PRS analyses for HTA. However, since it might benefit future studies interested in HTA, we conducted an exploratory GWAS (Table S6; Figures S8-S11).

\section{$3.5 \mid$ PRS}

PRS were tested for IQ, EA, and four neurodevelopmental conditions based on the behavioral correlations between $\mathrm{HT}$ and cognitive traits (Figure 2). PRS for ADHD showed an association with HT (Table 1 , Figure S12), indicating that higher genetic risk for ADHD is associated with higher $\mathrm{HT}$, that is, worse hearing. In contrast, schizophrenia PRS showed a negative association with $\mathrm{HT}$, suggesting that higher genetic risk for schizophrenia is associated with lower HT, that is, better hearing (Table 1, Figure S13). PRS for EA also reached significance for HT
(Table 1, Figure S14). The negative association suggests that a genetic liability toward higher EA is associated with better hearing.

We next tested whether the associations between ADHD and schizophrenia PRS with HT were mediated by cognitive skills and SES. We thus reran the PRS analysis on HT with ADHD and schizophrenia as training GWAS and using sex, the first two principal components, total IQ, GCSE, and SES as covariates. The effect of ADHD PRS on HT disappeared after adjusting for total IQ, GCSE, and SES (Table 1, Figure S15), suggesting that the association is mediated by these variables. In contrast, the effect of schizophrenia PRS on HT remained similar after adjusting for total IQ, GCSE, and SES (Table 1, Figure S16), suggesting that the association is not mediated by cognitive skills and SES.

\section{DISCUSSION}

We dissect the relationship between hearing measures and cognitive abilities and neurodevelopmental disorders both at the phenotypic and genetic level. We confirm that better hearing is associated with better performance on a range of cognitive tasks (Figure 2). We also report the results of the first GWAS on HT in children. Single marker, gene-based, and gene set enrichment analyses did not lead to any statistically significant results. However, PRS for EA and schizophrenia were significantly associated with HT (Table 1), with genetic liability for both traits associated with better hearing.

Phenotypic analysis for hearing measures showed sex-specific developmental effects for both HT and HTA. Our data ( $n=6,743$ ) show lower HT in boys compared to girls (Figure 1). The sex effect was very small and might only be detectable in reasonably sized samples, explaining why no sex effect was reported in a previous study of $n=1,869$ children and adolescents (Park et al., 2016). In adults, the reverse effect has been reported with better hearing in females compared to males (McFadden, 1993). In our data, HTA revealed an overall left ear advantage with a lower air conduction threshold of $0.28 \mathrm{~dB}$ on average (Figure 1), replicating the results from smaller studies in children (Rahko \& Karma, 1989; Roche et al., 1978). The effect was driven by females, suggesting that the sex effect on HTA reported in adults (i.e., stronger right ear advantage in males) seems to be established already in children. It is possible that a developmental shift toward the right ear in both sexes is driven by environmental factors, as continuous noise exposure (e.g., industrial noise) has been shown to affect the left ear more than the right (Nageris, Raveh, Zilberberg, \& Attias, 2007). A possible limitation of the current study is that stimulus presentation was not randomized, but the right ear was always tested first. Testing the right ear first has been more common in previous studies than vice versa, which could either result in a learning effect (favoring the left ear) or in a fatigue effect (favoring the right ear) (Pirilä, Jounio-Ervasti, \& Sorri, 1992). However, in adults, a right ear advantage is more common even in studies in which the order of stimulus presentation has been randomized (Pirilä et al., 1992), so the effect of stimulus presentation should be minimal. 
TAB LE 1 Results of PRS analysis on $\mathrm{HT}$

\begin{tabular}{|lrrrrr|}
\hline Trait/disorder & B & \multicolumn{1}{c}{ SE } & PRS $r^{2}$ (\%) & Full $\mathbf{r}^{2}$ (\%) & $p$ \\
\hline ADHD & 273.90 & 99.54 & 0.14 & 0.27 & .006 \\
\hline ASD & -14.63 & 8.03 & 0.06 & 0.19 & .068 \\
\hline Bipolar disorder & -43.26 & 29.10 & 0.04 & 0.17 & .137 \\
\hline Schizophrenia & -241.14 & 83.76 & 0.15 & 0.29 & .004 \\
\hline EA & $-1,564.72$ & 588.22 & 0.13 & 0.26 & .008 \\
\hline IQ & -41.93 & 28.45 & 0.04 & 0.17 & .141 \\
\hline $\begin{array}{l}\text { ADHD (corrected for total IQ, GCSE, } \\
\quad \text { SES) }\end{array}$ & 3.32 & 2.08 & 0.07 & 1.33 & .111 \\
\hline $\begin{array}{l}\text { Schizophrenia (corrected for total IQ, } \\
\text { GCSE, SES) }\end{array}$ & -274.08 & 103.66 & 0.20 & 1.45 & .008 \\
\hline
\end{tabular}

Consistent with previous studies (Moore et al., 2020), we found that in the normal range of variation, $\mathrm{HT}$ is negatively associated with several cognitive skills on the behavioral level (Figure 2). Although not significant, we found more negative HTA (i.e., more leftward asymmetry) in neurodevelopmental conditions including ASD compared to controls. Different types of asymmetry such as structural brain asymmetry (Postema et al., 2019), frontal alpha asymmetry (Gabard-Durnam, Tierney, VogelFarley, Tager-Flusberg, \& Nelson, 2015), language processing (Herringshaw, Ammons, DeRamus, \& Kana, 2016), and handedness (Markou, Ahtam, \& Papadatou-Pastou, 2017) have been implicated in ASD. Therefore, it might be worth collecting HTA measures more systematically to further investigate the link between asymmetries and disorders.

Because there was no significant SNP $h^{2}$ for HTA, downstream genomic analyses were only performed for HT. Although no single marker trait associations reached significance in the GWAS (Figure S4), the top marker on chromosome 15 (rs1039444) is located in an intron of $R A B 8 B$, which encodes for a GTPase that is expressed in inner and outer hair cells and is involved in autosomal recessive deafness (Heidrych et al., 2008). Targeted analysis for markers reported in previous GWAS for HT in adults replicated association with one marker, rs12955474, which is located in an intron of the CCBE1 gene (Fransen et al., 2015). Other markers in this gene have been associated with depression (Power et al., 2013) and left entorhinal cortex volume (Zhao et al., 2019). The sample size of our study is rather small for general GWAS standard, but of comparable size to GWAS conducted for hearing measures in adults previously. In fact, air conduction thresholds are not routinely collected in large-scale population studies. For example, the UK Biobank includes phenotypic information on hearing ability as self-reported hearing difficulty or use of hearing aids ( $n>300,000$ ) (Kalra et al., 2020; Wells et al., 2019). Similarly, a GWAS on age-related hearing loss in the Genetic Epidemiology Research on Adult Health and Aging (GERA) cohort $(n>50,000)$ used a case control design, identifying cases based on health records (ICD-9 diagnosis) (Hoffmann et al., 2016). GWASs on quantitative measures of hearing ability were limited to smaller sample sizes below 6,000 subjects for individual samples (Nolan et al., 2013; Vuckovic et al., 2015). Systematic collection of air conduction thresholds in both ears in children would enable larger genetic studies to dissect the links between hearing, cognition and neurodevelopment.
Genetic correlation analysis (Figure 3) revealed a tentative modest negative correlation between HT and reading ability. As lower HT indicate better hearing, this suggests an overlap of markers involved in better hearing and improved reading skills. In this analysis, standard errors were quite high, showcasing the need for follow up in larger datasets. We found no genetic correlation between verbal, performance or total IQ and HT. Similarly, PRS derived from large datasets did not identify an association between PRS for IQ (capturing mainly verbal and total IQ, Genç et al., 2021) and HT, suggesting that correlations we found on the behavioral level (Figure 2) are not mediated by shared biological pathways. Instead, the analysis of PRS for ADHD and schizophrenia support a role of the genetic risk for these neurodevelopmental disorders contributing to HT. Hearing deficits in ADHD have been reported in terms of speech perception (Fuermaier et al., 2018), but not in air conduction thresholds. In our sample, there was no association between HT and ADHD on the behavioral level; however, this analysis was based on a too small sample of children meeting the criteria for ADHD $(n=21)$ to make conclusive results (Table S2). Moreover, the effect of ADHD PRS on HT disappeared after adjusting for cognitive skills and SES, suggesting that the effect was mediated by cognitive factors.

Higher PRS for schizophrenia were associated with better hearing after adjustment for cognitive skills and SES. Most PRS studies report shared genetic risk for different outcomes, for example, risk for one disorder tends to increase risk for other disorders (Andlauer et al., 2021) or poor cognitive performance (Gialluisi et al., 2019). Instead, we found that PRS for schizophrenia were associated with better hearing. Similarly, PRS for schizophrenia have recently been associated with better language skills, but not overall school performance (Rajagopal et al., 2020). Therefore, our study expands the range of positive outcomes associated with risk of schizophrenia. The correlations between better hearing and cognitive skills (including language measures) found on the behavioral level (Figure 2) could be based on shared biological pathways which also increase the risk for schizophrenia. On the phenotypic level, previous research did not detect differences in HT between individuals affected by schizophrenia and controls in small samples ( $n=87)$ (Prager \& Jeste, 1993). In the future, this association might be worth being investigated more systematically in larger cohorts. 
In summary, our results highlight behavioral and genetic overlap between cognitive and sensory domains. We find that PRS for EA and schizophrenia are associated with HT, extending previous research reporting positive outcomes for schizophrenia PRS. Future studies should explore in more detail associations between sensory function and cognitive traits.

\section{ACKNOWLEDGMENTS}

The authors are extremely grateful to all the families who took part in this study, the midwives for their help in recruiting them, and the whole ALSPAC team, which includes interviewers, computer and laboratory technicians, clerical workers, research scientists, volunteers, managers, receptionists, and nurses. The authors are grateful to Veera M. Rajagopal for useful comments to the manuscript. The UK Medical Research Council and Wellcome (Grant ref: 217065/Z/19/Z) and the University of Bristol provide core support for ALSPAC. GWAS data were generated by Sample Logistics and Genotyping Facilities at Wellcome Sanger Institute and LabCorp (Laboratory Corporation of America) using support from 23andMe. This publication is the work of the authors and SP and JS will serve as guarantors for the analysis of the ALSPAC data presented in this article. JS is funded by the Deutsche Forschungsgemeinschaft (DFG, German Research Foundation, SCHM 3530/1-1, 418445085). SP is funded by the Royal Society (UF150663). Support to the genetic analysis was provided by the St Andrews Bioinformatics Unit funded by the Wellcome Trust [grant 105621/Z/14/Z].

\section{CONFLICT OF INTEREST}

The authors declare no competing financial interests in relation to the work described.

\section{DATA AVAILABILITY STATEMENT}

Data used for this submission will be made available on request to the ALSPAC Executive (alspac-exec@bristol.ac.uk). The ALSPAC data management plan (http://www.bristol.ac.uk/alspac/researchers/dataaccess/documents/alspac-data-management-plan.pdf) describes the data sharing policy, which is through a system of managed open access.

\section{ORCID}

Judith Schmitz (D) https://orcid.org/0000-0003-1031-0806

Silvia Paracchini (D) https://orcid.org/0000-0001-9934-8602

\section{REFERENCES}

Andlauer, T. F. M., Guzman-Parra, J., Streit, F., Strohmaier, J. González, M. J., Gil Flores, S., ... Rietschel, M. (2021). Bipolar multiplex families have an increased burden of common risk variants for psychiatric disorders. Molecular Psychiatry, 26(4), 1286-1298. https://doi. org/10.1038/s41380-019-0558-2

Autism Spectrum Disorders Working Group of The Psychiatric Genomics Consortium. (2017). Meta-analysis of GWAS of over 16,000 individuals with autism spectrum disorder highlights a novel locus at 10q24.32 and a significant overlap with schizophrenia. Molecular Autism, 8(1), 21. https://doi.org/10.1186/s13229-017-0137-9
Bishop, D. V. (1998). Development of the Children's communication checklist (CCC): A method for assessing qualitative aspects of communicative impairment in children. Journal of Child Psychology and Psychiatry, 39(6), 879-891.

Boyd, A., Golding, J., Macleod, J., Lawlor, D. A., Fraser, A., Henderson, J., ... Davey Smith, G. (2013). Cohort profile: The 'children of the 90s' - the index offspring of the Avon longitudinal study of parents and children. International Journal of Epidemiology, 42(1), 111-127. https://doi.org/ 10.1093/ije/dys064

Brandler, W. M., Morris, A. P., Evans, D. M., Scerri, T. S., Kemp, J. P., Timpson, N. J., ... Paracchini, S. (2013). Common variants in left/right asymmetry genes and pathways are associated with relative hand skill. PLoS Genetics, 9(9), e1003751. https://doi.org/10.1371/journal.pgen. 1003751

Chang, C. C., Chow, C. C., Tellier, L. C., Vattikuti, S., Purcell, S. M., \& Lee, J. J. (2015). Second-generation PLINK: Rising to the challenge of larger and richer datasets. GigaScience, 4, 7. https://doi.org/10.1186/ s13742-015-0047-8

Choi, S. M. R., Kei, J., \& Wilson, W. J. (2020). Learning difficulties and auditory processing deficits in a clinical sample of primary school-aged children. International Journal of Audiology, 59(11), 874-880. https://doi. org/10.1080/14992027.2020.1771782

Choi, S. W., \& O'Reilly, P. F. (2019). Prsice-2: Polygenic risk score software for biobank-scale data. GigaScience, 8(7), 1-6. https://doi.org/10. 1093/gigascience/giz082

Chung, D. Y., Mason, K., Gannon, R. P., \& Willson, G. N. (1983). The ear effect as a function of age and hearing loss. The Journal of the Acoustical Society of America, 73(4), 1277-1282. https://doi.org/10.1121/1. 389276

Combs, J. T. (2002). Lack of right ear advantage in patients with attentiondeficit/hyperactivity disorder. Clinical Pediatrics, 41(4), 231-234. https://doi.org/10.1177/000992280204100406

de Leeuw, C. A., Mooij, J. M., Heskes, T., \& Posthuma, D. (2015). Magma: Generalized gene-set analysis of GWAS data. PLoS Computational Biology, 11(4), e1004219. https://doi.org/10.1371/journal.pcbi.1004219

Diaz-Horta, O., Duman, D., Foster, J., Sirmac1, A., Gonzalez, M., Mahdieh, N., ... Tekin, M. (2012). Whole-exome sequencing efficiently detects rare mutations in autosomal recessive nonsyndromic hearing loss. PLoS One, 7(11), e50628. https://doi.org/10.1371/journal.pone. 0050628

Eagles, E. L. (1973). A longitudinal study of ear disease and hearing sensitivity in children. Audiology, 12(5), 438-445.

Felix, R. A., Gourévitch, B., \& Portfors, C. V. (2018). Subcortical pathways: Towards a better understanding of auditory disorders. Hearing Research, 362, 48-60. https://doi.org/10.1016/j.heares.2018.01.008

Fransen, E., Bonneux, S., Corneveaux, J. J., Schrauwen, I., Di Berardino, F., White, C. H., ... Friedman, R. A. (2015). Genome-wide association analysis demonstrates the highly polygenic character of age-related hearing impairment. European Journal of Human Genetics, 23(1), 110-115. https://doi.org/10.1038/ejhg.2014.56

Fraser, A., Macdonald-Wallis, C., Tilling, K., Boyd, A., Golding, J., Davey Smith, G., ... Lawlor, D. A. (2013). Cohort profile: The Avon longitudinal study of parents and children: Alspac mothers cohort. International Journal of Epidemiology, 42(1), 97-110. https://doi.org/10.1093/ije/ dys066

Friedman, R. A., van Laer, L., Huentelman, M. J., Sheth, S. S., van Eyken, E., Corneveaux, J. J., ... van Camp, G. (2009). Grm7 variants confer susceptibility to age-related hearing impairment. Human Molecular Genetics, 18(4), 785-796. https://doi.org/10.1093/hmg/ddn402

Fuermaier, A. B. M., Hüpen, P., de Vries, S. M., Müller, M., Kok, F. M., Koerts, J., ... Tucha, O. (2018). Perception in attention deficit hyperactivity disorder. Attention Deficit and Hyperactivity Disorders, 10(1), 2147. https://doi.org/10.1007/s12402-017-0230-0

Gabard-Durnam, L., Tierney, A. L., Vogel-Farley, V., Tager-Flusberg, H., \& Nelson, C. A. (2015). Alpha asymmetry in infants at risk for autism 
spectrum disorders. Journal of Autism and Developmental Disorders, 45(2), 473-480. https://doi.org/10.1007/s10803-013-1926-4

Gates, G. A., Couropmitree, N. N., \& Myers, R. H. (1999). Genetic associations in age-related hearing thresholds. Archives of OtolaryngologyHead \& Neck Surgery, 125(6), 654-659. https://doi.org/10.1001/ archotol.125.6.654

Gathercole, S. E., Willis, C. S., Baddeley, A. D., \& Emslie, H. (1994). The Children's test of nonword repetition: A test of phonological working memory. Memory, 2(2), 103-127. https://doi.org/10.1080/ 09658219408258940

Genç, E., Schlüter, C., Fraenz, C., Arning, L., Metzen, D., Nguyen, H. P., ... Ocklenburg, S. (2021). Polygenic scores for cognitive abilities and their association with different aspects of general intelligence-a deep phenotyping approach. Molecular Neurobiology, 58, 4145-4156. https://doi.org/10.1007/s12035-021-02398-7

Gialluisi, A., Andlauer, T. F. M., Mirza-Schreiber, N., Moll, K., Becker, J., Hoffmann, P., ... Schulte-Körne, G. (2019). Genome-wide association scan identifies new variants associated with a cognitive predictor of dyslexia. Translational Psychiatry, 9(1), 77. https://doi.org/10.1038/ s41398-019-0402-0

Girotto, G., Pirastu, N., Sorice, R., Biino, G., Campbell, H., d'Adamo, A. P., ... Gasparini, P. (2011). Hearing function and thresholds: A genome-wide association study in European isolated populations identifies new loci and pathways. Journal of Medical Genetics, 48(6), 369-374. https://doi. org/10.1136/jmg.2010.088310

Gruzelier, J. H., \& Hammond, N. V. (1979). Gains, losses and lateral differences in the hearing of schizophrenic patients. British Journal of Psychology, 70(2), 319-330. https://doi.org/10.1111/j.2044-8295.1979. tb01689.x

Håvik, B., Degenhardt, F. A., Johansson, S., Fernandes, C. P. D., Hinney, A., Scherag, A., ... Le Hellard, S. (2012). Dclk1 variants are associated across schizophrenia and attention deficit/hyperactivity disorder. PLoS One, 7(4), e35424. https://doi.org/10.1371/journal. pone.0035424

Heidrych, P., Zimmermann, U., Bress, A., Pusch, C. M., Ruth, P., Pfister, M., ... Blin, N. (2008). Rab8b GTPase, a protein transport regulator, is an interacting partner of otoferlin, defective in a human autosomal recessive deafness form. Human Molecular Genetics, 17(23), 3814-3821. https://doi.org/10.1093/hmg/ddn279

Herringshaw, A. J., Ammons, C. J., DeRamus, T. P., \& Kana, R. K. (2016). Hemispheric differences in language processing in autism spectrum disorders: A meta-analysis of neuroimaging studies. Autism Research, 9(10), 1046-1057. https://doi.org/10.1002/aur.1599

Hoffmann, T. J., Keats, B. J., Yoshikawa, N., Schaefer, C., Risch, N., \& Lustig, L. R. (2016). A large genome-wide association study of agerelated hearing impairment using electronic health records. PLoS Genetics, 12(10), e1006371. https://doi.org/10.1371/journal.pgen. 1006371

Huyghe, J. R., van Laer, L., Hendrickx, J.-J., Fransen, E., Demeester, K., Topsakal, V., ... van Camp, G. (2008). Genome-wide SNP-based linkage scan identifies a locus on 8q24 for an age-related hearing impairment trait. American Journal of Human Genetics, 83(3), 401-407. https://doi. org/10.1016/j.ajhg.2008.08.002

James, A. L., \& Barry, R. J. (1983). Developmental effects in the cerebral lateralization of autistic, retarded, and normal children. Journal of Autism and Developmental Disorders, 13(1), 43-56. https://doi.org/10. 1007/BF01531358

Kakarlapudi, V., Sawyer, R., \& Staecker, H. (2003). The effect of diabetes on sensorineural hearing loss. Otology \& Neurotology, 24(3), 382-386. https://doi.org/10.1097/00129492-200305000-00006

Kalra, G., Milon, B., Casella, A. M., Herb, B. R., Humphries, E., Song, Y., ... Ament, S. A. (2020). Biological insights from multi-omic analysis of 31 genomic risk loci for adult hearing difficulty. PLoS Genetics, 16(9), e1009025. https://doi.org/10.1371/journal.pgen.1009025
Kannan, P. M., \& Lipscomb, D. M. (1974). Letter: Bilateral hearing asymmetry in a large population. The Journal of the Acoustical Society of America, 55(5), 1092-1094. https://doi.org/10.1121/1.1914657

Karlsson, K. K., Harris, J. R., \& Svartengren, M. (1997). Description and primary results from an audiometric study of male twins. Ear and Hearing, 18(2), 114-120. https://doi.org/10.1097/00003446-19970400000003

Khalfa, S., Bruneau, N., Rogé, B., Georgieff, N., Veuillet, E., Adrien, J. L., ... Collet, L. (2001). Peripheral auditory asymmetry in infantile autism. The European Journal of Neuroscience, 13(3), 628-632. https://doi.org/ 10.1046/j.1460-9568.2001.01423.x

Khalfa, S., \& Collet, L. (1996). Functional asymmetry of medial olivocochlear system in humans. Towards a peripheral auditory lateralization. Neuroreport, 7(5), 993-996. https://doi.org/10.1097/ 00001756-199604100-00008

Lee, J. J., Wedow, R., Okbay, A., Kong, E., Maghzian, O., Zacher, M., ... Cesarini, D. (2018). Gene discovery and polygenic prediction from a genome-wide association study of educational attainment in 1.1 million individuals. Nature Genetics, 50(8), 1112-1121. https://doi.org/ 10.1038/s41588-018-0147-3

Loh, P.-R., Kichaev, G., Gazal, S., Schoech, A. P., \& Price, A. L. (2018). Mixed-model association for biobank-scale datasets. Nature Genetics, 50(7), 906-908. https://doi.org/10.1038/s41588-018-0144-6

Loh, P.-R., Tucker, G., Bulik-Sullivan, B. K., Vilhjálmsson, B. J., Finucane, H. K., Salem, R. M., ... Price, A. L. (2015). Efficient Bayesian mixed-model analysis increases association power in large cohorts. Nature Genetics, 47(3), 284-290. https://doi.org/10.1038/ng.3190

Markou, P., Ahtam, B., \& Papadatou-Pastou, M. (2017). Elevated levels of atypical handedness in autism: Meta-analyses. Neuropsychology Review, 27(3), 258-283. https://doi.org/10.1007/s11065-017-9354-4

Mathew, V. M., Gruzelier, J. H., \& Liddle, P. F. (1993). Lateral asymmetries in auditory acuity distinguish hallucinating from nonhallucinating schizophrenic patients. Psychiatry Research, 46(2), 127-138. https:// doi.org/10.1016/0165-1781(93)90015-9

McFadden, D. (1993). A speculation about the parallel ear asymmetries and sex differences in hearing sensitivity and otoacoustic emissions. Hearing Research, 68(2), 143-151. https://doi.org/10.1016/03785955(93)90118-k

Moore, D. R., Zobay, O., \& Ferguson, M. A. (2020). Minimal and mild hearing loss in children: Association with auditory perception, cognition, and communication problems. Ear and Hearing, 41(4), 720-732. https://doi.org/10.1097/AUD.0000000000000802

Morata, T. C. (2003). Chemical exposure as a risk factor for hearing loss. Journal of Occupational and Environmental Medicine, 45(7), 676-682. https://doi.org/10.1097/01.jom.0000071507.96740.70

Nageris, B. I., Raveh, E., Zilberberg, M., \& Attias, J. (2007). Asymmetry in noise-induced hearing loss: Relevance of acoustic reflex and left or right handedness. Otology \& Neurotology, 28(4), 434-437. https://doi. org/10.1097/mao.0b013e3180430191

Neale, B. M., Medland, S. E., Ripke, S., Asherson, P., Franke, B., Lesch, K.P., ... Nelson, S. (2010). Meta-analysis of genome-wide association studies of attention-deficit/hyperactivity disorder. Journal of the American Academy of Child and Adolescent Psychiatry, 49(9), 884-897. https://doi.org/10.1016/j.jaac.2010.06.008

Newbury, D. F., Gibson, J. L., Conti-Ramsden, G., Pickles, A., Durkin, K., \& Toseeb, U. (2019). Using polygenic profiles to predict variation in language and psychosocial outcomes in early and middle childhood. Journal of Speech, Language, and Hearing Research, 62(9), 3381-3396. https://doi.org/10.1044/2019_JSLHR-L-19-0001

Newbury, D. F., Winchester, L., Addis, L., Paracchini, S., Buckingham, L.-L., Clark, A., ... Monaco, A. P. (2009). Cmip and ATP2C2 modulate phonological short-term memory in language impairment. American Journal of Human Genetics, 85(2), 264-272. https://doi.org/10.1016/j.ajhg.2009. 07.004 
Nolan, L. S., Maier, H., Hermans-Borgmeyer, I., Girotto, G., Ecob, R., Pirastu, N., ... Dawson, S. J. (2013). Estrogen-related receptor gamma and hearing function: Evidence of a role in humans and mice. Neurobiology of Aging, 34(8), 2077.e1-9. https://doi.org/10.1016/j. neurobiolaging.2013.02.009

Park, Y. H., Shin, S.-H., Byun, S. W., \& Kim, J. Y. (2016). Age- and genderrelated mean hearing threshold in a highly-screened population: The Korean National Health and Nutrition Examination Survey 2010-2012. PLoS One, 11(3), e0150783. https://doi.org/10.1371/ journal.pone.0150783

Pirilä, T., Jounio-Ervasti, K., \& Sorri, M. (1992). Left-right asymmetries in hearing threshold levels in three age groups of a random population. Audiology, 31(3), 150-161. https://doi.org/10.3109/ 00206099209072910

Postema, M. C., van Rooij, D., Anagnostou, E., Arango, C., Auzias, G. Behrmann, M., ... Francks, C. (2019). Altered structural brain asymmetry in autism spectrum disorder in a study of 54 datasets. Nature Communications, 10(1), 4958. https://doi.org/10.1038/s41467-01913005-8

Power, R. A., Cohen-Woods, S., Ng, M. Y., Butler, A. W., Craddock, N., Korszun, A., ... Uher, R. (2013). Genome-wide association analysis accounting for environmental factors through propensity-score matching: Application to stressful live events in major depressive disorder. American Journal of Medical Genetics. Part B, Neuropsychiatric Genetics, 162B(6), 521-529. https://doi.org/10.1002/ajmg.b.32180

Prager, S., \& Jeste, D. V. (1993). Sensory impairment in late-life schizophrenia. Schizophrenia Bulletin, 19(4), 755-772. https://doi.org/10. 1093/schbul/19.4.755

Psychiatric GWAS Consortium Bipolar Disorder Working Group. (2011). Large-scale genome-wide association analysis of bipolar disorder identifies a new susceptibility locus near ODZ4. Nature Genetics, 43(10), 977-983. https://doi.org/10.1038/ng.943

Rahko, T., \& Karma, P. (1989). Pure-tone hearing thresholds in otologically healthy 5-year-old children in Finland. Archives of Oto-Rhino-Laryngology, 246(3), 137-141. https://doi.org/10.1007/BF00456654

Rajagopal, V. M., Ganna, A., Coleman, J. R. I., Allegrini, A. G., Voloudakis, G., Grove, J., ... Demontis, D. (2020). Genome-wide association study of school grades identifies a genetic overlap between language ability, psychopathology and creativity. BioRxiv. https://doi.org/ 10.1101/2020.05.09.075226

Rashid, V., Engberink, M. F., van Eijsden, M., Nicolaou, M., Dekker, L. H., Verhoeff, A. P., \& Weijs, P. J. M. (2018). Ethnicity and socioeconomic status are related to dietary patterns at age 5 in the Amsterdam born children and their development (ABCD) cohort. BMC Public Health 18(1), 115. https://doi.org/10.1186/s12889-017-5014-0

Reite, M., Teale, P., Rojas, D. C., Reite, E., Asherin, R., \& Hernandez, O. (2009). Meg auditory evoked fields suggest altered structural/functional asymmetry in primary but not secondary auditory cortex in bipolar disorder. Bipolar Disorders, 11(4), 371-381. https:// doi.org/10.1111/j.1399-5618.2009.00701.x

Roche, A. F., Siervogel, R. M., Himes, J. H., \& Johnson, D. L. (1978). Longitudinal study of hearing in children: Baseline data concerning auditory thresholds, noise exposure, and biological factors. The Journal of the Acoustical Society of America, 64(6), 1593-1616. https://doi.org/10. 1121/1.382143

Ruhl, D. S., Cable, B. B., \& Martell, D. W. (2014). Medication associated with hearing loss: 25 years of medical malpractice cases in the United States. Otolaryngology-Head and Neck Surgery, 151(3), 431437. https://doi.org/10.1177/0194599814536850

Rust, J. (1996). WOLD Wechsler objective language dimensions manual. London, England: Psychological Corp.

Rust, J., Golombok, S., \& Trickey, G. (1993). WORD Wechsler objective Reading dimensions manual. Sidcup, England: Psychological Corp.

Savage, J. E., Jansen, P. R., Stringer, S., Watanabe, K., Bryois, J., de Leeuw, C. A., ... Posthuma, D. (2018). Genome-wide association meta- analysis in 269,867 individuals identifies new genetic and functional links to intelligence. Nature Genetics, 50(7), 912-919. https://doi.org/ 10.1038/s41588-018-0152-6

Scerri, T. S., Morris, A. P., Buckingham, L.-L., Newbury, D. F., Miller, L. L., Monaco, A. P., ... Paracchini, S. (2011). Dcdc2, KIAA0319 and CMIP are associated with reading-related traits. Biological Psychiatry, 70(3), 237-245. https://doi.org/10.1016/j.biopsych.2011.02.005

Schizophrenia Working Group of the Psychiatric Genomics Consortium. (2014). Biological insights from 108 schizophrenia-associated genetic loci. Nature, 511(7510), 421-427. https://doi.org/10.1038/ nature13595

Schmidt, G. L., Rey, M. M., Oram Cardy, J. E., \& Roberts, T. P. L. (2009). Absence of M100 source asymmetry in autism associated with language functioning. Neuroreport, 20(11), 1037-1041. https://doi.org/ 10.1097/WNR.0b013e32832e0ca7

Schultz, J. M., Bhatti, R., Madeo, A. C., Turriff, A., Muskett, J. A., Zalewski, C. K., ... Friedman, T. B. (2011). Allelic hierarchy of CDH23 mutations causing non-syndromic deafness DFNB12 or Usher syndrome USH1D in compound heterozygotes. Journal of Medical Genetics, 48(11), 767-775. https://doi.org/10.1136/jmedgenet-2011100262

Schwander, M., Kachar, B., \& Müller, U. (2010). Review series: The cell biology of hearing. The Journal of Cell Biology, 190(1), 9-20. https:// doi.org/10.1083/jcb.201001138

Stanbury, M., Rafferty, A. P., \& Rosenman, K. (2008). Prevalence of hearing loss and work-related noise-induced hearing loss in Michigan. Journal of Occupational and Environmental Medicine, 50(1), 72-79. https://doi. org/10.1097/JOM.0b013e31815b568c

Stevenson, J., Pimperton, H., Kreppner, J., Worsfold, S., Terlektsi, E., Mahon, M., \& Kennedy, C. (2018). Language and reading comprehension in middle childhood predicts emotional and behaviour difficulties in adolescence for those with permanent childhood hearing loss. Journal of Child Psychology and Psychiatry, 59(2), 180-190. https://doi.org/ 10.1111/jcpp.12803

Takaki, H., Kikuta, R., Shibata, H., Ninomiya, H., Tashiro, N., \& Fukumaki, Y. (2004). Positive associations of polymorphisms in the metabotropic glutamate receptor type 8 gene (GRM8) with schizophrenia. American Journal of Medical Genetics. Part B, Neuropsychiatric Genetics, 128B(1), 6-14. https://doi.org/10.1002/ajmg.b.20108

Veuillet, E., Georgieff, N., Philibert, B., Dalery, J., Marie-Cardine, M., \& Collet, L. (2001). Abnormal peripheral auditory asymmetry in schizophrenia. Journal of Neurology, Neurosurgery, and Psychiatry, 70(1), 8894. https://doi.org/10.1136/jnnp.70.1.88

Viljanen, A., Era, P., Kaprio, J., Pyykkö, I., Koskenvuo, M., \& Rantanen, T. (2007). Genetic and environmental influences on hearing in older women. The Journals of Gerontology. Series A, Biological Sciences and Medical Sciences, 62(4), 447-452. https://doi.org/10.1093/gerona/62.4.447

Viljanen, A., Kaprio, J., Pyykkö, I., Sorri, M., Kauppinen, M., Koskenvuo, M., \& Rantanen, T. (2007). Genetic and environmental influences on hearing at different frequencies separately for the better and worse hearing ear in older women. International Journal of Audiology, 46(12), 772-779. https://doi.org/10.1080/ 14992020701581422

Vuckovic, D., Dawson, S., Scheffer, D. I., Rantanen, T., Morgan, A., Di Stazio, M., ... Girotto, G. (2015). Genome-wide association analysis on normal hearing function identifies PCDH20 and SLC28A3 as candidates for hearing function and loss. Human Molecular Genetics, 24(19), 5655-5664. https://doi.org/10.1093/hmg/ddv279

Wang, K., Li, M., \& Hakonarson, H. (2010). Annovar: Functional annotation of genetic variants from high-throughput sequencing data. Nucleic Acids Research, 38(16), e164. https://doi.org/10.1093/nar/gkq603

Warren, E. H., \& Liberman, M. (1989). Effects of contralateral sound on auditory-nerve responses. I. Contributions of cochlear efferents. Hearing Research, 37(2), 89-104. https://doi.org/10.1016/0378-5955(89) 90032-4 
Watanabe, K., Taskesen, E., van Bochoven, A., \& Posthuma, D. (2017). Functional mapping and annotation of genetic associations with FUMA. Nature Communications, 8(1), 1826. https://doi.org/10.1038/ s41467-017-01261-5

Wechsler, D., Golombok, S., \& Rust, J. (1991). Wechsler intelligence scale for children - third edition UKmanual. Sidcup, England: Psychological Corp.

Wells, H. R. R., Freidin, M. B., Zainul Abidin, F. N., Payton, A., Dawes, P., Munro, K. J., ... Williams, F. M. K. (2019). Gwas identifies 44 independent associated genomic loci for self-reported adult hearing difficulty in UKbiobank. American Journal of Human Genetics, 105(4), 788-802. https://doi.org/10.1016/j.ajhg.2019.09.008

Wolber, L. E., Girotto, G., Buniello, A., Vuckovic, D., Pirastu, N., LorenteCánovas, B., ... Williams, F. M. K. (2014). Salt-inducible kinase 3, SIK3, is a new gene associated with hearing. Human Molecular Genetics, 23(23), 6407-6418. https://doi.org/10.1093/hmg/ddu346

Yang, J., Benyamin, B., McEvoy, B. P., Gordon, S., Henders, A. K., Nyholt, D. R., ... Visscher, P. M. (2010). Common SNPs explain a large proportion of the heritability for human height. Nature Genetics, 42(7), 565-569. https://doi.org/10.1038/ng.608

Yang, J., Lee, S. H., Goddard, M. E., \& Visscher, P. M. (2011). Gcta: A tool for genome-wide complex trait analysis. American Journal of Human Genetics, 88(1), 76-82. https://doi.org/10.1016/j.ajhg.2010. 11.011
Zhang, L., Zhong, X., An, Z., Han, S., Luo, X., Shi, Y., \& Yi, Q. (2014). Association analysis of the GRM8 gene with schizophrenia in the Uygur Chinese population. Hereditas, 151(6), 140-144. https://doi. org/10.1111/hrd2.00045

Zhao, B., Luo, T., Li, T., Li, Y., Zhang, J., Shan, Y., ... Zhu, H. (2019). Genome-wide association analysis of 19,629 individuals identifies variants influencing regional brain volumes and refines their genetic coarchitecture with cognitive and mental health traits. Nature Genetics, 51(11), 1637-1644. https://doi.org/10.1038/s41588-019-0516-6

\section{SUPPORTING INFORMATION}

Additional supporting information may be found in the online version of the article at the publisher's website.

How to cite this article: Schmitz, J., Abbondanza, F., \& Paracchini, S. (2021). Genome-wide association study and polygenic risk score analysis for hearing measures in children. American Journal of Medical Genetics Part B: Neuropsychiatric Genetics, 1-11. https://doi.org/10.1002/ajmg.b.32873 